\title{
Revisión sistemática de la resistencia antimicrobiana en cocos Gram positivos intrahospitalarios en Colombia
}

\author{
Carmelo José Espinosa1', Jorge Alberto Cortés ${ }^{1,2}$, Juan Sebastián Castillo², Aura Lucía Leal ${ }^{2,3}$ \\ 1 Departamento de Medicina, Facultad de Medicina, Universidad Nacional de Colombia, Bogotá, D.C., Colombia \\ 2 Grupo de Investigación en Enfermedades Infecciosas, Universidad Nacional de Colombia, Bogotá, D.C., \\ Colombia \\ ${ }^{3}$ Departamento de Microbiología, Facultad de Medicina, Universidad Nacional de Colombia, Bogotá, D.C., \\ Colombia
}

Introducción. La resistencia bacteriana es un problema de salud pública a nivel mundial, cuyo adecuado manejo implica el conocimiento de su presencia y comportamiento en cada uno de los países y regiones del mundo.

Objetivos. Describir el perfil de resistencia a los distintos antimicrobianos marcadores en microorganismos Gram positivos identificados en hospitales colombianos.

Materiales y métodos. Se realizó una revisión sistemática de la literatura indexada en Medline y Lilacs, además de la búsqueda manual de todos los números en revistas colombianas reconocidas y afines a la infectología para identificar referencias no disponibles electrónicamente.

Resultados. De 34 estudios observacionales, sólo se cuenta con reportes consecutivos en años a partir del 2001, estos principalmente para Bogotá. La tasa de resistencia a la meticilina de Staphylococcus aureus y estafilococos coagulasa negativos en Bogotá, de aislamientos en servicios diferentes a la unidad de cuidados intensivos, oscilan de $35 \%$ a $50 \%$ y de $72 \%$ a $76 \%$, respectivamente; en aislamientos de la unidad de cuidados intensivos, la resistencia osciló de $35 \%$ a $71 \%$ y de $74 \%$ a 83 $\%$, respectivamente. La tasa de resistencia a vancomicina para Enterococcus faecium en Bogotá es menor de $20 \%$ con variaciones muy grandes con el paso de los años.

Conclusiones. Hay una alta resistencia a los antibióticos marcadores en los aislamientos de Gram positivos identificados en hospitales de las principales ciudades colombianas.

Palabras clave: farmacorresistencia microbiana, pruebas de sensibilidad microbiana, vigilancia sanitaria, Staphylococcus, Enterococcus.

Systematic review of antimicrobial resistance among Gram positive cocci in hospitals in Colombia

Introduction. Bacterial resistance is a public health problem worldwide whose proper management requires knowledge of its presence and its behavior in each region and country.

Objectives. A survey of the medical literature was conducted to identify levels of resistance to antibiotic markers in Gram positive bacterial isolates from Colombian hospitals.

Materials and methods. A systematic review of the literature included articles indexed in MEDLINE and LILACS. A manual search was made of Colombian scientific journals and other infectious disease literature not available electronically.

Results. A total of 34 observational studies were located, including a series of consecutive reports initiated in 2001. Most of the reports came from the city of Bogota. The rate of methicillin resistance for Staphylococcus aureus and coagulase-negative staphylococci in non intensive care unit isolates ranged between $35 \%-50 \%$ and $72 \%-76 \%$, respectively. Resistance in intensive care unit isolates had a range between $35 \%-71 \%$ and $74 \%-83 \%$, respectively. The rate of vancomycin-resistant Enterococcus faecium averaged less than $20 \%$ over the years but with large annual variation .

Conclusions. Resistance markers appeared in high frequency among Gram positive isolates identified in hospitals in major Colombian cities.

Key words: Drug resistance, microbial; microbial sensitivity tests, health surveillance, Staphylococcus, Enterococcus. 
En los últimos 20 años la resistencia a los antimicrobianos a nivel mundial ha aumentado de manera significativa, hasta el punto de que la Organización Mundial de la Salud (OMS) en 1999 catalogó el fenómeno como un problema de salud pública. Desde entonces, se ha iniciado una gran cantidad de medidas para su control, las cuales incluyen generar información sobre los perfiles de resistencia para cada una de las regiones y países $(1,2)$.

Las bacterias Gram positivas, particularmente Staphylococcus aureus, estafilococos coagulasa negativos y Enterococcus spp., representan patógenos de gran importancia en el medio ambiente hospitalario. Datos basados de estudios epidemiológicos de redes de vigilancia en países desarrollados, entre ellos los Estados Unidos, muestran que hasta $60 \%$ de las infecciones del torrente sanguíneo son causadas por este grupo de gérmenes, y que la seguridad terapéutica de los esquemas de antibióticos disponibles se ve amenazada por la emergencia de cepas resistentes, las cuales muestran un incremento progresivo con el paso de los años $(3,4)$.

A finales de la década pasada se registraron los primeros reportes de vigilancia de la resistencia bacteriana en Colombia y en los años recientes el interés en este campo ha generado un incremento progresivo de información, con la consolidación de grupos de investigación y de redes de información organizada que han permitido el estudio del fenómeno en nuestro medio. Existe un reconocido compromiso institucional con la divulgación de reportes epidemiológicos de resistencia bacteriana en el ámbito local, así como reportes de algunas iniciativas de vigilancia; sin embargo, no existe a la fecha una síntesis de las tasas reportadas en la literatura nacional para estos microorganismos.

En el presente estudio se reportan los hallazgos de una revisión sistemática de la literatura sobre los perfiles de resistencia bacteriana en microorganismos intrahospitalarios Gram positivos, la cual incluye información publicada o presentada en eventos científicos.

\footnotetext{
Correspondencia:

Jorge Alberto Cortés, Facultad de Medicina, oficina 510, Universidad Nacional de Colombia, Ciudad Universitaria, Bogotá, D.C., Colombia.

Teléfono: (571) 316 5000, extensión 15011

jorgecortes@yahoo.com

Recibido: 16/02/10; aceptado:17/09/10
}

\section{Materiales y métodos}

Se condujo una revisión sistemática de la literatura publicada sobre la resistencia bacteriana a nivel hospitalario de Gram positivos en Colombia, entre enero de 1980 y diciembre de 2008. En ella se incluyeron las bases de datos Medline, Embase y Lilacs, además de la búsqueda manual en los registros de publicación de todas las revistas biomédicas colombianas incluyendo las publicaciones científicas de congresos afines al tema, adecuando los lineamientos de la colaboración Cochrane para la realización de revisiones sistemáticas (5).

Se diseñaron búsquedas acordes a cada una de las bases de datos. En el caso de Medline, se utilizó la siguiente estructura de búsqueda: ((Resist $\left.{ }^{\star}\right)$ AND (antimicrob* OR bacterial OR antibiot*)) AND (Colombia OR medell* OR bogot* OR Cali OR bucaraman* OR Monter* OR cartagena OR Barranquilla). No se consideraron restricciones de idioma ni de fecha de publicación. Los marcadores epidemiológicos de relevancia clínica que fueron evaluados en el presente estudio son $S$. aureus resistente a la meticilina, Enterococcus spp. resistente a la vancomicina y estafilococos coagulasa negativos con patrón de resistencia a la meticilina.

Los artículos fueron seleccionados inicialmente de acuerdo con la información consignada en su título y resumen. Los criterios de inclusión fueron: estudios observacionales analíticos (cohortes, casos y controles, corte transversal) y descriptivos (estudios ecológicos, series y reporte de casos, reportes de vigilancia) que reportaran porcentajes de resistencia de aislamientos provenientes de laboratorios institucionales en hospitales colombianos; estos reportes debían contener información completa y adecuada sobre los perfiles de resistencia para cada uno de los antibióticos considerados de relevancia clínica.

Se excluyeron los estudios sobre aislamientos por conveniencia, (por ejemplo, pruebas de antibióticos para mercadeo) y aquellos estudios provenientes de aislamientos exclusivos en pediatría y los estudios considerados como reportes de mala calidad o incongruentes.

Luego de la recolección de los estudios, dos evaluadores-ciegos e independientes-determinaron para cada uno de ellos en su formato de texto completo la presencia de los criterios para la inclusión o exclusión del análisis final. 
Para la extracción de la información se dispuso de un formato con especial énfasis en el tipo de estudio, metodología, resultados y evaluación global. Se hizo una descripción de los hallazgos, consolidando la información de los artículos que resultaron relevantes y que fueron considerados de buena calidad a juicio de los investigadores, junto con el reporte de los porcentajes de resistencia para cada uno de los marcadores.

Finalmente, se determinó la distribución de los perfiles de resistencia por aéreas clínicas relevantes, como cuidados intensivos y el resto de áreas hospitalarias. Los resultados sobre resistencia bacteriana se reportaron como porcentaje de resistencia para cada antibiótico de importancia. Cuando se disponía de información redundante (más de un estudio sobre un área geográfica y un tipo de sala), se expresó la información en rangos inferiores y superiores de las tasas de resistencia.

Además, los resultados se categorizaron según el área hospitalaria en la cual se realizó el aislamiento: aquellos procedentes de la unidad de cuidados intensivos y todos aquellos no procedentes de ésta, es decir, de la sala general de hospitalización.

\section{Resultados}

Se identificaron 36 artículos a través de buscadores en internet y 78 a través de la búsqueda manual, incluyendo 10 estudios que fueron identificados por medio de las dos metodologías.

De los 104 estudios potenciales, 52 eran originales, un número igual eran resúmenes de congresos y 2 eran boletines epidemiológicos. De los resúmenes excluidos (72 reportes), 61 reportes tenían información incompleta o irrelevante sobre el tema de estudio, 4 daban información exclusiva para pediatría y 7 correspondían a estudios en los cuales se buscaban aislamientos por conveniencia.

En total se incluyeron 33 estudios observacionales sobre el perfil de resistencia a los antimicrobianos de bacterias Gram positivas para el análisis final (6-19) (cuadro 1). De estos 33 estudios, 12 se encontraban publicados en revistas con evaluación pareada, 2 eran boletines y 19 habían sido presentados como resúmenes en congresos de investigación. De los 12 manuscritos en revistas, 9 habían sido publicados en revistas colombianas y 3 en revistas internacionales.

De todos los estudios identificados, 33 estudios informaron datos para el marcador $S$. aureus resistente a la meticilina, 16 para el marcador estafilococos coagulasa negativos, 10 para Enterococcus faecium y 6 para Enterococcus faecalis.

De los estudios incluidos, 14 correspondían a aislamientos exclusivos de Bogotá, 4 de Montería, 3 de Medellín, 2 de Caldas, 2 de Cali y 9 estudios reportaron aislamientos simultáneos de varias ciudades en el país $(7,12-14)$.

No se encontraron reportes sobre resistencia bacteriana antes de 1993 y sólo a partir de 2001 se tienen reportes consecutivos.

La tasa de resistencia a meticilina para los aislamientos de $S$. aureus en Bogotá se encuentra reportada en un estudio aislado para 1996; posteriormente, varios estudios permiten obtener los datos consecutivos anualmente a partir de 2001 (6-11). Los porcentajes mínimos y máximos de resistencia por año se pueden observar en la figura 1 para aislamientos provenientes de la unidad de cuidados intensivos y fuera de ella. El porcentaje de resistencia a la meticilina para todo tipo de aislamiento hospitalario en otras ciudades, se encuentra reportada para algunos años en la figura 2 .

La tasa de resistencia a la meticilina en el marcador de estafilococos coagulasa negativos en Bogotá se encuentra reportada para todos los años a partir del 2001. De manera característica, los porcentajes fluctúan entre $72 \%$ y $76 \%$ para cualquiera de los años en aislamientos procedentes fuera de la unidad de cuidados intensivos y entre $78 \%$ y 87 $\%$, para los aislamientos procedentes de dicha unidad (cuadro 2). Medellín reporta una tasa de $61 \%$ para 1996 (11), mientras que Cali reporta $74 \%$ para 1997 para cualquier tipo de aislamiento hospitalario.

La resistencia a la vancomicina fue examinada de manera independiente por los estudios para las cepas de E. faecalis y E. faecium. De manera característica, los estudios para este tipo de gérmenes provienen de Bogotá y como reporte de resistencia en Colombia por aislamientos en múltiples ciudades del país $(7,12-14)$. La tasa de resistencia para cada uno de los años y por áreas hospitalarias se puede observar en el cuadro 2.

\section{Discusión}

La resistencia bacteriana constituye un problema importante para los servicios de atención sanitaria. Desde sus inicios alrededor de la década de 1940 ha mostrado un incremento progresivo, 
Cuadro 1. Estudios incluidos en la revisión sistemática ${ }^{€}$.

\begin{tabular}{|c|c|c|c|c|c|}
\hline \multirow{2}{*}{ Estudio y referencia } & \multirow[b]{2}{*}{ Periodo } & \multirow[b]{2}{*}{ Ciudad } & \multirow[b]{2}{*}{$\begin{array}{c}\text { Lugar } \\
\text { hospitalario }\end{array}$} & \multicolumn{2}{|c|}{ Aislamientos } \\
\hline & & & & $\begin{array}{c}\text { Gram } \\
\text { positivos }\end{array}$ & Totales \\
\hline Leal AL et al. (6) & $2001-2003$ & Bogotá & Todos & 13.148 & 84.664 \\
\hline Arias CA (7) & $2001-2002$ & Colombia & Todos & 296 & 597 \\
\hline Robledo J (Resumen I 1, VI Congreso & & & & & \\
\hline Colombiano de Enfermedades Infecciosas, 2003) & $2001-2002$ & Medellín & Todos & ND & 18.554 \\
\hline $\begin{array}{l}\text { Crespo MP (Resumen I5, VI Congreso Colombiano } \\
\text { de Enfermedades Infecciosas, 2003) }\end{array}$ & $1999-2002$ & Cali & Externos & ND & 2.165 \\
\hline $\begin{array}{l}\text { Leal AL (Resumen I 6, VI Congreso Colombiano } \\
\text { de Enfermedades Infecciosas, 2003) }\end{array}$ & $2001-2002$ & Bogotá & No UCl & ND & 39.728 \\
\hline Vesga O (8) & $1987-1993$ & Medellín & Todos & 53 & 53 \\
\hline Castañeda CR (Resumen 75, XIV Congreso & & & & & \\
\hline Colombiano de Medicina Interna, 1996) & $1995-1996$ & Cali & Todos & 11 & 88 \\
\hline Arango A. (Resumen 74, XIV Congreso & & & & & \\
\hline Colombiano de Medicina Interna, 1996) & $1995-1996$ & Bogotá & $\mathrm{UCl}$ & ND & ND \\
\hline Álvarez C (9) & $2001-2003$ & Bogotá & $\mathrm{UCl}$ & 2.560 & 18.407 \\
\hline Cortés J (10) & $2001-2005$ & Bogotá & Externos & 2.308 & 2.308 \\
\hline Buitrago G ( Resumen G 25, IV Encuentro de & & & & & \\
\hline Enfermedades Infecciosas, 2004) & $2002-2003$ & Bogotá & Todos & 65 & 272 \\
\hline Robledo J (11) & $1997-1997$ & Medellín & Todos & 30 & 212 \\
\hline $\begin{array}{l}\text { Osorio L (Resumen F 12, VI Congreso Colombiano } \\
\text { de Enfermedades Infecciosas, 2003) }\end{array}$ & $2002-2002$ & Bogotá & Todos & 535 & 1.428 \\
\hline Reyes J (12) & $2001-2003$ & Colombia & Todos & 348 & 2.325 \\
\hline Rosenthal VD (13) & $2002-2005$ & Colombia & $\mathrm{UCl}$ & ND & ND \\
\hline Hidalgo M (14) & $2001-2003$ & Colombia & Todos & 348 & 917 \\
\hline Jaramillo E (15) & $1992-1994$ & Caldas & $\mathrm{UCl}$ & 158 & 1718 \\
\hline Bermúdez DC (Resumen C 15, V Encuentro de & & & & & \\
\hline Enfermedades Infecciosas, 2006) & 2003-2004 & Bogotá & Todos & ND & ND \\
\hline $\begin{array}{l}\text { Buitrago G (Resumen B 9, V Encuentro de } \\
\text { Enfermedades Infecciosas, 2006) }\end{array}$ & & & & & \\
\hline $\begin{array}{l}\text { Entermedades Intecclosas, } 2006 \text { ) } \\
\text { Jaramillo AC (Resumen B 11, V Encuentro de }\end{array}$ & $2001-2005$ & Bogota & UCT & 1193 & $91 / 9$ \\
\hline enfermedades infecciosas, 2006) & 2003-2004 & Colombia & Todos & 146 & 146 \\
\hline Leal AL (Resumen B 13, V Encuentro de & & & & & \\
\hline Enfermedades Infecciosas, 2006) & $2001-2005$ & Bogotá & $\mathrm{UCl}$ & ND & 139.615 \\
\hline $\begin{array}{l}\text { Buitrago G. (Resumen A 29, VI Encuentro de } \\
\text { enfermedades infecciosas, 2008) }\end{array}$ & $2001-2006$ & Bogotá & Todos & 33.156 & 33.156 \\
\hline $\begin{array}{l}\text { Martínez R (Resumen C 71, VI Encuentro de } \\
\text { Enfermedades Infecciosas, 2008) }\end{array}$ & $2007-2008$ & Montería & Todos & ND & 590 \\
\hline $\begin{array}{l}\text { Reyes J. (Resumen A 80, VI Encuentro de } \\
\text { enfermedades infecciosas, 2008) }\end{array}$ & $2006-2008$ & Colombia & Todos & 707 & 707 \\
\hline Rodríguez F (Resumen A 81, VI Encuentro de & & & & & \\
\hline Enfermedades Infecciosas, 2008) & $2005-2007$ & Colombia & $\mathrm{UCl}$ & 2071 & 14796 \\
\hline Panesso D (Resumen A 85, VI Encuentro de & & & & & \\
\hline Enfermedades Infecciosas, 2008) & $2006-2008$ & Colombia & Todos & 40 & 309 \\
\hline $\begin{array}{l}\text { Raciny M. (Resumen C 190, VI Encuentro de } \\
\text { enfermedades infecciosas, 2008) }\end{array}$ & $2005-2007$ & Montería & Todos & 23 & 23 \\
\hline Espinal P (Resumen C 235, VI Encuentro de & & & & & \\
\hline Enfermedades Infecciosas, 2008) & $2005-2007$ & Colombia & Todos & 23 & 23 \\
\hline Secretaría Distritalo de Salud (16) & 2006 & Bogotá & No UCI & 4.463 & 30.947 \\
\hline GREBO (17) & $2001-2008$ & Bogotá & $\mathrm{UCl}$ & 617 & 5.269 \\
\hline Martínez P (18) & $2001-2002$ & Montería & Todos & 67 & 283 \\
\hline Pérez D (19) & 2003-2003 & Montería & Todos & 13 & 144 \\
\hline $\begin{array}{l}\text { Robayo DB (Resumen G 20, III Encuentro de } \\
\text { Enfermedades Infecciosas, 2002) }\end{array}$ & $2001-2002$ & Caldas & Todos & 25 & 58 \\
\hline
\end{tabular}

${ }^{€}$ Unidad de cuidados intensivos (UCl); externos corresponde a aislamientos de pacientes ambulatorios; ND: datos no disponibles 


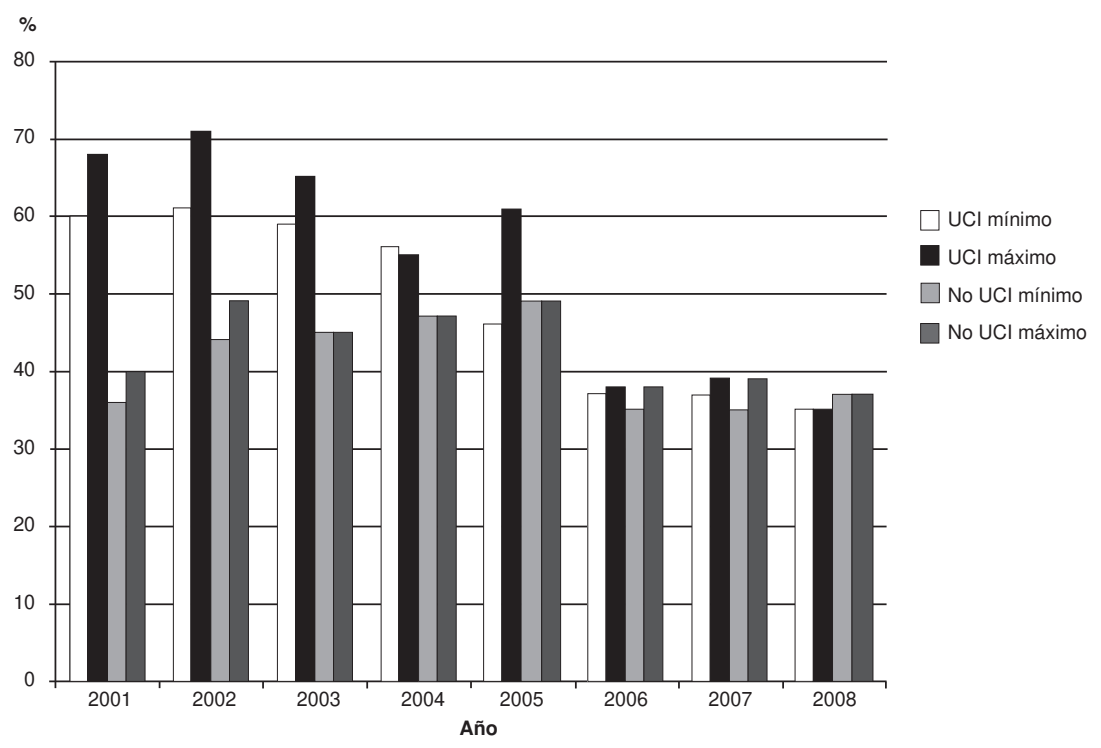

Figura 1. Porcentaje de aislamientos identificados como Staphylococcus aureus resistente a la meticilina en unidades de cuidados intensivos y en salas diferentes a las de cuidados intensivos en Bogotá. Fuente: Leal AL. Resumen B13. V Encuentro de Enfermedades Infecciosas 2006 (16,17).

continuo y sin fronteras, abarcando todas las naciones del mundo $(4,5,20)$. Su emergencia ha constituido un problema central para la vigilancia en salud desde la década de 1980, cuando a nivel mundial se empezó a reportar la presencia de gérmenes hospitalarios resistentes que lograban predominancia en el desarrollo de infecciones de origen fundamentalmente hospitalario $(4,5,20)$. Desde entonces, los países desarrollados crearon las primeras redes organizadas de vigilancia en el tema para conocer el fenómeno y, a partir de allí, plantear e implementar soluciones agrupadas bajo la denominación de "uso racional de antibióticos" (21) y, en los últimos años, el término antibiotic stewardship (22).

En Colombia, al igual que en la mayoría de los países en vía de desarrollo, el problema seguramente emerge simultáneamente y en magnitudes que usualmente se desconocen ante la ausencia de estudios de buena calidad diseñados de manera específica para ello. Nuestra investigación encontró que los primeros estudios publicados en el país datan de $1993(8,15)$ y que sólo en 2002 se consolidó la primera red de vigilancia organizada para el estudio del tema en la ciudad de Bogotá (6). Sin embargo, en la actualidad el número de ciudades e instituciones involucradas en el seguimiento del fenómeno ha aumentado, al igual que el número de publicaciones por años. Entre las ciudades destacadas en este proceso encontramos a Bogotá, Cali, Medellín y Montería (6-8,12-14).

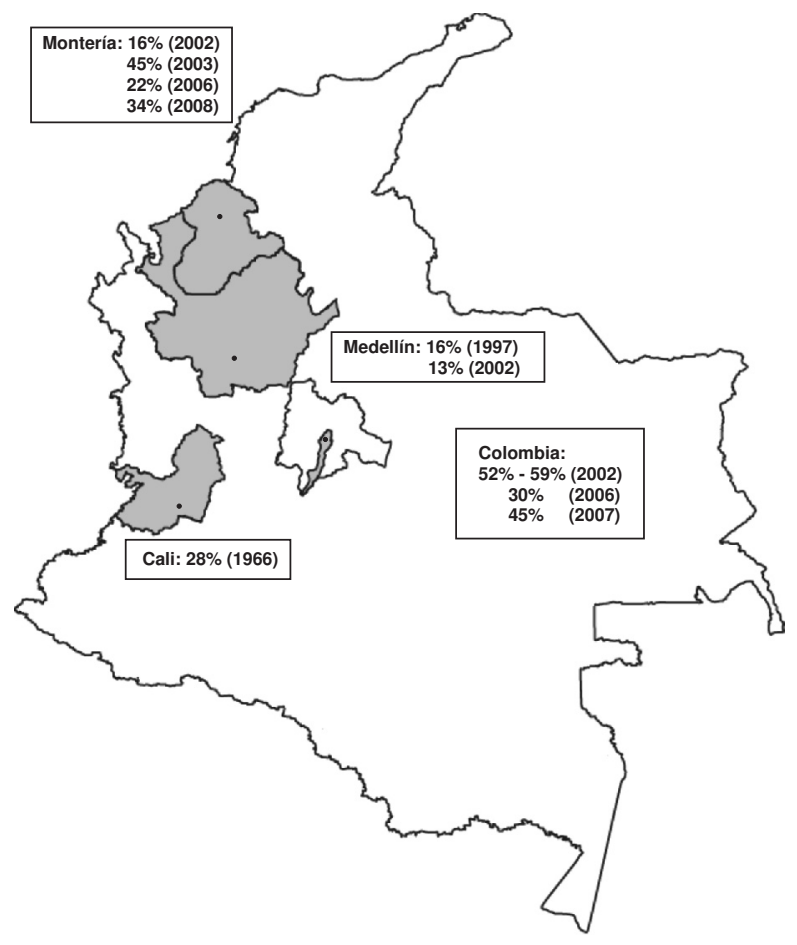

Figura a partir de datos de: Jaramillo AC (Resumen B 11, V Encuentro de Enfermedades Infecciosas, 2006), Martínez R (Resumen C 71, VI Encuentro de Enfermedades Infecciosas, 2008), Castañeda CR (Resumen 75, XIV Congreso Colombiano de medicina interna, 1996), Panesso D (Resumen A 85, VI Encuentro de Enfermedades Infecciosas, 2008), Raciny M (Resumen C 190, VI Encuentro de Enfermedades Infecciosas, 2008), Espinal P (Resumen C 235, VI Encuentro de Enfermedades Infecciosas, 2008) $(11,26,27)$.

Colombia, corresponde con aislamientos simultáneo en varias ciudades en el país.

Figura 2. Porcentaje de Staphylococcus aureus resistente a la meticilina en todo tipo de aislamiento hospitalario en ciudades colombianas, en diferentes años y por área geográfica. 
Cuadro 2. Porcentaje de resistencia en aislamientos de estafilococos coagulasa negativos, Enterococcus faecalis y Enterococcus faecium en Bogotá por área hospitalaria y por año.

\begin{tabular}{|c|c|c|c|c|c|c|c|c|c|c|c|c|c|c|c|c|}
\hline \multirow[t]{2}{*}{ Germen } & \multicolumn{2}{|c|}{2001} & \multicolumn{2}{|r|}{2002} & \multicolumn{2}{|c|}{2003} & \multicolumn{2}{|r|}{2004} & \multicolumn{2}{|c|}{2005} & \multicolumn{2}{|c|}{2006} & \multicolumn{2}{|c|}{2007} & \multicolumn{2}{|c|}{2008} \\
\hline & UCI & No UCl & UCl & No UCl & $\mathrm{UCl}$ & No UCl & UCl & No UCl & UCl & No UCI & UCl & No UCI & UCl & No UCl & $\mathrm{UCl}$ & No UCI \\
\hline $\begin{array}{l}\mathrm{ECN}^{*} \\
\text { Enterococcus }\end{array}$ & $83-87$ & $75-75$ & $79-80$ & $72-72$ & $78-81$ & $73-73$ & $79-79$ & $73-73$ & $82-82$ & $73-73$ & $77-80$ & $72-76$ & $74-81$ & $72-76$ & $78-78$ & $76-76$ \\
\hline $\begin{array}{l}\text { faecium }^{\star *} \\
\text { Enterococcus }^{*}\end{array}$ & $12-15$ & $15-17$ & $17-18$ & $5-5$ & $0-10$ & $4-4$ & $1-1$ & $3-3$ & $1-10$ & $3-3$ & $2-3$ & $2-3$ & $2-3$ & $2-2$ & $20-20$ & $17-17$ \\
\hline faecalis ${ }^{* *}$ & $2-2$ & $1-1$ & $4-6$ & $1-1$ & $3-5$ & $2-2$ & $7-7$ & $3-3$ & $2-2$ & $2-2$ & $0-1$ & $1-1$ & $0-1$ & $1-1$ & $1-1$ & $1-1$ \\
\hline
\end{tabular}

* El marcador de resistencia en estafilococos coagulasa negativos es la meticilina.

** El marcador de resistencia en las especies de Enterococcus fue la vancomicina.

Cuadro con datos de (9), G. Buitrago (Resumen G 25, IV Encuentro de Enfermedades Infecciosas, 2004), G. Buitrago (Resumen B 9, V Encuentro de Enfermedades Infecciosas, 2006), A. L. Leal (Resumen B 13, V Encuentro de Enfermedades Infecciosas, 2006) $(16,17)$.

Las infecciones del torrente sanguíneo son causadas principalmente por gérmenes Gram positivos, entre los cuales $S$. aureus, los estafilococos coagulasa negativos y las especies de Enterococcus corresponden a los más importantes. En países como Estados Unidos, la tasa de resistencia a la meticilina para las cepas de $S$. aureus ha sufrido un incremento progresivo con el paso de los años. Por ejemplo, para los aislamientos procedentes de la unidad de cuidados intensivos se reportó una tasa de $59 \%$ para 2005 , lo cual significa un aumento de $11 \%$ con respecto a la frecuencia de resistencia reportada para $2002(4,20)$. Esta tasa se mantuvo estable (57\%) para el período de 2006 a 2007. Además, la tasa de resistencia a este marcador puede tener pequeñas variaciones y encontrarse entre $49 \%$ en las infecciones del sitio operatorio, y $65 \%$, en las infecciones urinarias asociadas con la sonda vesical (23). Asimismo, el fenómeno se presenta en los aislamientos de especies de enterococos, en los cuales la tasa de resistencia a la vancomicina se elevó de $17 \%$ en 1999 a $25 \%$ en el $2004(4,21)$.

Países sociodemográficamente similares al nuestro, como Brasil, reportaron una tasa de $S$. aureus resistente a la meticilina para todo tipo de asilamiento hospitalario de $44 \%$ en 2001 y de $79 \%$ para los estafilococos coagulasa negativos. Nuestros países vecinos, Ecuador y Venezuela, informaron a la Organización Panamericana de la Salud (OPS) en el 2004 una tasa de resistencia inferior a la identificada en nuestro país para ese momento (25\% Vs. $47 \%$ ) (24). La tasa de resistencia a la vancomicina en aislamientos de especies de Enterococcus para el mismo periodo estaba alrededor de $7 \%$ en Brasil (25). En nuestro país el problema se eleva a niveles similares a los de Brasil, en donde la frecuencia de $S$. aureus resistente a la meticilina es mucho más alta para los aislamientos de la unidad de cuidados intensivos, con los máximos valores para la Bogotá entre 60 $\%$ y $70 \%$, para el periodo de 2001 al 2003. Estas cifras parecen haber disminuido con el paso de los años, a un nivel estable de $35 \%$ en el período del 2008.

Para los aislamientos que no provienen de la unidad de cuidados intensivos, la tasa de resistencia muestra un patrón más estable, alrededor de 40 $\%$ a $50 \%$, en todo el periodo de 2001 a 2008. A diferencia de otros países, la tasa de resistencia a la vancomicina en los aislamientos de la unidad de cuidados intensivo para el periodo de 2004 a 2005 era muy baja, menor de $10 \%$, con un aumento notorio a $20 \%$ en 2008. Este comportamiento puede estar relacionado con la presencia de brotes en hospitales específicos.

Resulta difícil comparar la información de diferentes países con diferentes sistemas de salud y diferentes estrategias de vigilancia. Además, es posible que algunas de las diferencias observadas en los países estén relacionadas con la distribución de los clones de $S$. aureus en diferentes regiones. América Latina se puede dividir en dos regiones: aquéllas con clones brasileños y aquellas sin este tipo de clones (26). Colombia comparte con Chile, Paraguay y Uruguay esta última característica, mientras se desconoce la situación de nuestros vecinos Ecuador y Venezuela.

En la actualidad se acepta que la resistencia a un determinado antibiótico se encuentra baja cuando sus valores son inferiores a $10 \%$; en ese sentido, el uso rutinario de dicho antibiótico en el cubrimiento empírico de una infección está justificado $(2,27)$.

Respecto de las infecciones por gérmenes Gram positivos, los resultados de nuestra investigación muestran que el panorama es bastante desalentador y que el fenómeno, al igual que en otros países, se presenta en nuestros servicios de atención sanitaria en una magnitud importante. Ello implica que en 
fenómenos infecciosos del torrente sanguíneo causados por gérmenes de este tipo se indique como primera opción terapéutica la vancomicina y que, probablemente, otros fenómenos infecciosos hospitalarios, como los asociados a la piel, también tengan este tipo de consideración, lo que, además, se sostiene en la ausencia de reportes en el país de cepas de $S$. aureus resistentes a la vancomicina.

Respecto a las infecciones abdominales asociadas a cepas de Enterococcus, la ampicilina sigue siendo la mejor opción terapéutica, y la resistencia a la vancomicina muestra patrones por brotes que requieren básicamente de una mejoría en las medidas del control de los aislamientos (7). Sin embargo, vale la pena mencionar que la mayor parte de la información no depende de análisis moleculares que permitan confirmar la especie de Enterococcus identificada, lo cual constituye un sesgo en lo información presentada.

En el presente estudio se recopila la información producto de la investigación en resistencia bacteriana en el país, la cual muestra un aumento en su interés con el paso de los años. La mayoría de los estudios encontrados reportan un número limitado de aislamientos, no todos están basados en redes organizadas de vigilancia en el tema, con procedimientos estandarizados de detección y reporte, y con variedad en los métodos usados para su desarrollo. Existe la posibilidad de que los estudios reportados no hagan uso de aislamientos consecutivos y totales en un periodo.

Todo lo anterior determina una gran limitación de los resultados encontrados y, de manera especial, dificultad para que los investigadores muestren las tendencias de los porcentajes de resistencia; es decir, como se ve en los resultados, no es posible afirmar que exista una tendencia a la disminución de los porcentajes de resistencia con el paso de los años. Los estudios simultáneos en redes locales de vigilancia organizada están procurando generar este tipo de información. Dada la naturaleza de la información evaluada, fue necesario adaptar los estándares de revisión disponibles para otros tipos de estudios; estas diferencias suponen limitaciones a la hora de evaluar aspectos como los sesgos de publicación.

La mayor utilidad de la presente investigación está en establecer un punto de partida y resumir la información con que disponemos en Colombia, derivada de la investigación en este campo en los años recientes. El esfuerzo continuado y organizado de los sistemas de vigilancia permitirá conocer mejor el comportamiento del fenómeno y evaluar los esfuerzos continuados que se han venido gestando en las instituciones y en el sistema de salud. Los esfuerzos para unificar las redes de vigilancia son bienvenidos, ya que permitirán unificar criterios y estrategias de búsqueda, así como causar un impacto en el manejo clínico de estas infecciones y reforzar las estrategias efectivas para la prevención, como el lavado de manos.

\section{Agradecimientos}

A la estudiante María Amelia Bueno, quien colaboró en la recolección de la información.

\section{Conflicto de intereses}

No hay conflictos de interés que señalar por parte de los autores.

\section{Financiación}

Este estudio fue financiado mediante un apoyo de la División de Investigación de la sede Bogotá de la Universidad Nacional de Colombia, programa de apoyo a tesis de posgrado, código 7649.

\section{Referencias}

1. Tenover FC. Mechanisms of antimicrobial resistance in bacteria. Am J Infect Control. 2006;34:S3-10.

2. Kaye KS, Engemann JJ, Fraimow HS y Abrutyn E. Pathogens resistant to antimicrobial agents: Epidemiology, molecular mechanisms, and clinical management. Infect Dis Clin North Am. 2004;18:467-511.

3. Rybak MJ. Pharmacodynamics: Relation to antimicrobial resistance. Am J Infect Control. 2006;34:S38-45.

4. Rice LB. Antimicrobial resistance in gram-positive bacteria. Am J Infect Control. 2006;34:S11-9.

5. Higgins JP, Green S. Cochrane handbook for systematic reviews of interventions 4.2.6. Fecha de consulta: 6 de octubre de 2006. Disponible en: http://www.cochrane.org/ resources/handbook/hbook.htm.

6. Leal AL, Eslava-Schmalbach J, Álvarez C, Buitrago G, Méndez M. Endemic tendencies and bacterial resistance markers in third-level hospitals in Bogota, Colombia. Rev Salud Pública (Bogotá). 2006;8(Suppl.1):59-70.

7. Arias CA, Reyes J, Zúniga M, Cortés L, Cruz C, Rico CL, et al. Multicentre surveillance of antimicrobial resistance in enterococci and staphylococci from Colombian hospitals, 2001-2002. J Antimicrob Chemother. 2003;51:59-68.

8. Vesga O, Toro JM. Sepsis por Staphylococcus aureus. Acta Med Colomb. 1994;19:116-24.

9. Álvarez C, Cortés J, Arango A, Correa C, Leal A. Antimicrobial resistance in intensive care units in Bogotá, Colombia, 2001-2003. Rev Salud Pública (Bogotá). 2006;8(Suppl.1):86-101.

10. Cortés JA, Gómez CA, Cuervo SI, Leal AL. Communityacquired methicillin-resistant Staphylococcus aureus in 
Bogotá, Colombia: Public health implications. Rev Salud Pública (Bogotá). 2007;9:448-54.

11. Robledo J, López J, Sierra P, Vesga O, Pfaller M. El programa de vigilancia antimicrobiana SENTRY en Colombia, hallazgos iniciales en tres hospitales de Medellín. Infectio. 1999;3:448-54.

12. Reyes J, Hidalgo M, Díaz L, Rincón S, Moreno J, Vanegas $\mathbf{N}$, et al. Characterization of macrolide resistance in Grampositive cocci from Colombian hospitals: A countrywide surveillance. Int J Infect Dis. 2007;11:329-36.

13. Rosenthal VD, Maki DG, Salomao R, Moreno CA, Mehta Y, Higuera F, et al. Device-associated nosocomial infections in 55 intensive care units of 8 developing countries. Ann Intern Med. 2006;145:582-91.

14. Hidalgo M, Reyes J, Cárdenas AM, Díaz L, Rincón S, Vanegas $\mathbf{N}$, et al. Resistance profiles to fluoroquinolones in clinical isolates of Gram positive cocci. Biomédica. 2008;28:284-94.

15. Jaramillo EL. Resistencia bacteriana a los antibióticos en la Unidad de Cuidados Intensivos, Hospital de Caldas, 1992-1994. Colombia Médica. 1996;27:69-76.

16. Secretaría de Salud de Bogotá. Boletín epidemiológico distrital de infecciones intrahospitalarias 2007. Bogotá D.C.: Secretaría de Salud de Bogotá; 2007. p. 1-15.

17. Grupo para el Control de la Resistencia Bacteriana GREBO. Boletín informativo GREBO 2008. Bogotá D.C.: Gráficas Ducal; 2008.

18. Martínez $\mathbf{P}$, Mercado M, Mattar S. Actividad in vitro de moxifloxacina comparada con otros antibióticos frente a aislamientos nosocomiales de dos hospitales de Colombia. Universitas Medicas. 2004;45:101-9.

19. Pérez D, Mattar S, Mercado M. High resistance of nosocomial germs in Monteria, Colombia. Universitas Medicas. 2003;44:131-7.
20. Shorr AF. Epidemiology of staphylococcal resistance. Clin Infect Dis. 2007;45(Suppl.3):S171-6.

21. Amyes SG. Enterococci and streptococci. Int J Antimicrob Agents. 2007;29(Suppl.3):S43-52.

22. Dellit TH, Owens RC, McGowan JE Jr, Gerding DN, Weinstein RA, Burke JP, et al. Infectious Diseases Society of America and the Society for Healthcare Epidemiology of America guidelines for developing an institutional program to enhance antimicrobial stewardship. Clin Infect Dis. 2007;44:159-77.

23. Hidron Al, Edwards JR, Patel J, Horan TC, Sievert DM, Pollock DA, et al. NHSN annual update: Antimicrobialresistant pathogens associated with healthcare-associated infections: Annual summary of data reported to the National Healthcare Safety Network at the Centers for Disease Control and Prevention, 2006-2007. Infect Control Hosp Epidemiol. 2008;29:996-1011.

24. Guzmán-Blanco $\mathbf{M}$, Mejía $\mathbf{C}$, Isturiz $\mathbf{R}$, Álvarez $\mathbf{C}$, Bavestrello L, Gotuzzo E, et al. Epidemiology of methicillinresistant Staphylococcus aureus (MRSA) in Latin America. Int J Antimicrob Agents. 2009;34:304-8.

25. Sader HS, Jones RN, Gales AC, Silva JB, Pignatari AC. SENTRY antimicrobial surveillance program report: Latin American and Brazilian results for 1997 through 2001. Braz J Infect Dis. 2004;8:25-79.

26. Rodríguez-Noriega E, Seas C, Guzmán-Blanco M, Mejía C, Álvarez C, Bavestrello L, et al. Evolution of methicillinresistant Staphylococcus aureus clones in Latin America. Int J Infect Dis. 2010;14:e560-6.

27. Livermore DM. Minimising antibiotic resistance. Lancet Infect Dis. 2005;5:450-9. 